\title{
Treatment for Severe Pregnancy Induced Hypertension and Preterm La- bor with Concomitant Administration of Estradiol and Progesterone under Monitoring Aminopeptidases: A Case Report
}

\section{Mizutani $\mathbf{S}^{1}$, Mizutani $\mathrm{E}^{1}$, Iwase $\mathrm{A}^{2}$ and Shibata $\mathrm{K}^{\mathbf{3}^{*}}$}

${ }^{1}$ Department of Obstetrics and Gynecology, Daiya Building Ladys Clinic, Meieki, Nakamura-ku, Nagoya, Japan ${ }^{2}$ Department of Obstetrics and Gynecology, Gunnma University Graduate School of Medicine, Takasaki, Japan ${ }^{3}$ Department of Obstetrics and Gynecology, Banbuntane Hotokukai Hospital, Fujita Health University, Nagoya, Japan

\begin{abstract}
The knowledge for molecular mechanisms of preterm labor and preeclampsia is still premature. In the previous review, the author proposed that sex steroid treatment should be conserved for prospective clinical study in both preterm labor and preeclampsia. Unfortunately, little attention has been paid so far on the review article. Here, the authors present two cases of severe, complicated preeclampsia associated with preterm labor.
\end{abstract}

Keywords: $\quad$ Estradiol-progesterone therapy; Placental aminopeptidases (APs); Angiotensin II (A-II); Vasopressin (AVP); Oxytocin (OT)

\section{Introduction}

Underlying mechanism of preeclampsia and onset of labor/preterm labor, despite progress in its research on molecular basis, need to be elucidated until effective treatments are established. Historically, peptide hormones such as angiotensin II (A-II), vasopressin (AVP) and oxytocin (OT), have been investigated as essential hormones involved in preeclampsia and onset of labor/preterm labor but recently scientists' effort towards these hormones has somehow declined these days. It is important to look at these hormones in contrast to their degrading enzymes.

The authors cloned placental leucine aminopeptidase (P-LAP), the enzyme that effectively degrades AVP and OT. Serum concentrations of P-LAP increase in pregnant women as pregnancy advances $[1,2]$. The authors have shown existence of aminopeptidase A (APA) in human placenta as an identical substance to the A-II degrading enzyme. Later, Max Cooper cloned APA as a B lymphocyte differentiation antigen. The authors reported the fact that APA increases in maternal sera in accordance with gestational term in clinical settings [1,3]. These peptide hormones are known essential for the development of fetus and thus increase with fetal development. Concentration of these hormones in the feto-placental unit is higher than those of maternal circulation. Since the hormones are biologically active, the leak of these hormones into the maternal circulation is strictly regulated by degradation activity of placental aminopeptidases (APs) in order to maintain maternal blood pressure (BP) normal and to keep uterine tonus under control. If the fetal secretion of A-II and AVP were increased, following the the stress such as hypoxia, the leak of these fetal hormones into maternal circulation can results in the increase of BP and uterine contraction. Previous studies by the authors indicated both maternal P-LAP and APA activities became significantly higher in mild preeclampsia than those of normal pregnancy: suggesting protective actions of P-LAP and APA against the leak of fetal hormones into maternal circulation [2-5].

It has been clarified therefore that, P-LAP and APA activities in pregnancy sera are tightly linked to preeclampsia and preterm labor: their activities shall decrease into lower levels compared to normal pregnancy with worsening of the condition. The clinical cases shown in this report suggest underlying dynamics of fetal hormones through changes in the degradation activities of APs in preeclampsia and preterm labor [1-4]. Besides sex steroids: estradiol and progesterone
(P), in pregnancy sera also increase similarly as APs with advancing of normal gestation. Serum concentrations of these hormones are like the levels of placental APs, not only in normal pregnancy but also in preeclampsia and preterm labor. Dynamics of APs and sex steroids in maternal sera is therefore essentially similar to each other in both normal and complicated pregnancies [1].

In 1940, Smith \& Smith reported a favorable outcome of the treatment by using estradiol benzoate (E2) accompanied with $\mathrm{P}$ in severe preeclampsia. In 1969, taking a hint from Smith's case reports, the author had tried his own, first, sex steroids treatment in severe preeclampsia on a dose increase by gestational week method, about measuring P-LAP levels [5,6]. Owing to alleviation of clinical symptoms, blood pressure and natriuresis, prolongation of gestation at least for 3 weeks was witnessed, and the treatment was proved successful. In view of neither safe nor effective medication being practiced at present, the proposed sex steroid treatment in this report seems worthwhile for the prospective controlled studies for the treatment of both preeclampsia and preterm labor [6].

The knowledge for molecular mechanisms of preterm labor and preeclampsia is still premature. Moreover, clinical effectiveness of $\mathrm{P}$ and 17-ahydroxyprogesterone capronate (17-P) in preterm labor remains under debate [7]. In the previous review, the author proposed that sex steroid treatment should be conserved for prospective clinical study in both preterm labor and preeclampsia. Unfortunately, little attention has been paid so far on the review article. Here, the authors present two cases of severe, complicated preeclampsia associated with preterm labor.

\section{Case Presentation}

\section{Case 1}

The patient, 39-year-old woman (gravid-1, para-0) was conceived

${ }^{*}$ Corresponding author: Shibata K, Department of Obstetrics and Gynecology, Banbuntane Hotokukai Hospital, Fujita Health University, Nagoya, Japan, Tel: +81-52-321-8171; E-mail: shiba@med.nagoya-u.ac.jp

Received May 16, 2018; Accepted May 21, 2018; Published May 25, 2018

Citation: Mizutani S, Mizutani E, Iwase A, Shibata K (2018) Treatment for Severe Pregnancy Induced Hypertension and Preterm Labor with Concomitant Administration of Estradiol and Progesterone under Monitoring Aminopeptidases: A Case Report. J Clin Case Rep 8: 1115. doi: 10.4172/2165-7920.10001115

Copyright: @ 2018 Mizutani S, et al. This is an open-access article distributed under the terms of the Creative Commons Attribution License, which permits unrestricted use, distribution, and reproduction in any medium, provided the original author and source are credited. 
Citation: Mizutani S, Mizutani E, Iwase A, Shibata K (2018) Treatment for Severe Pregnancy Induced Hypertension and Preterm Labor with Concomitant Administration of Estradiol and Progesterone under Monitoring Aminopeptidases: A Case Report. J Clin Case Rep 8: 1115. doi: 10.4172/2165-7920.10001115

Page 2 of 5

by in vitro fertilization frozen embryo transfer (IVF-ET) in 2003. Her pregnancy was a di-chorionic twin. Until this pregnancy she was without any medical complication. Her pregnancy had been uneventful until week 22, however at week 26, she was hospitalized due to severe preeclampsia (BP 190/108, proteinuria and edema) complicated with premature labor. She was treated by intravenous drip injection of beta-2 agonist (ritodorine), and intramuscular injection of terbutaline $(2 \mathrm{mg}$ twice/day), in addition, with indomethacin $(50 \mathrm{mg})$ to control uterine contraction.

Both nifedipine $(40 \mathrm{mg} /$ day $)$ and metoprolol $(80 \mathrm{mg} /$ day $)$ were used concomitantly as antihypertensives. However, these agents will pass through the placenta easily thereby can exert negative effects on the fetus, against its well-being. The estimated fetal weight of twin A was $900 \mathrm{~g}$ and twin B was $750 \mathrm{~g}$, respectively at the hospital admission. At week 27+3-day clinical symptoms had not improved and the non-stressed test (NST) showed the decreased variability and late decelerations observed. At this stage, she was transferred to our hospital. On admission, the estimated fetal weight of twin A was $1050 \mathrm{~g}$ without ominous sign, on the other hand, twin B was $850 \mathrm{~g}$ and this fetus showed the reversed umbilical blood flow by Pulse Doppler Assessment, suggesting ominous sign.

Firstly, we stopped all medicines used in the previous hospital except ritodorine. We explained her and her family about the anticipated poor prognosis of her fetuses (especially twin B), even if interruption of her pregnancy was tried by cesarean section at this stage. We proposed her our sex steroid treatment accompanied by ritodorine in order to carry over her pregnancy and avoid preterm delivery. The patient and her family accepted our proposal.

We started the sex steroid treatment with ritodorine on the day of admission: E2 and P injections daily according to the doses corresponded to advancing gestational stages shown in our review article [6]. Next morning, the intra-uterine twin B death was confirmed by cardiotachometer. Since this case is a dichorionic twin, negative outcomes, such as twin-twin transfusion syndrome were thought unlikely. Rarely has it been reported that single fetal deaths may induce release of fibrin and thromboplastin in circulation leading to DIC [8]. Nevertheless we monitored the changes of coagulation markers: fibrinogen, D-dimer and FDP periodically during her pregnancy. Both D-dimer and FDP levels were high before the death of twin B, but after the death, these markers had decreased and thereafter did not change. Fibrinogen levels kept constant throughout her pregnancy irrespective of the death of twin B (data not shown).

On this sex steroid treatment, without using any antihypertensive agent, diuretics, nor magnesium sulfate, the patient's BP showed the decreasing tendency to fall as in Figure 1 and finally to lower than 150 mmHg. In the week 31 we could discontinue ritodorine because the uterine contraction had disappeared. Figure 2 shows the changes of P-LAP activities in maternal sera since the admission to our hospital. Until the death of twin B, P-LAP activities were more or less in the range of normal twin pregnancy referred by Mizutani, et al. [9] but kept monitored if its activity levels showed a tendency to fall [10]. BP decreased temporarily but thereafter it became normal. A good condition of twin A was achieved after the sex steroid treatment (Figure 1). APA activity levels in maternal sera continued at lower levels than mean normal pregnancy [2], nevertheless, a constant tendency toward increase with sex steroid treatment was observed (data not shown).

Because the patient's BP had normalized, steroids doses were lowered at week $33+5$ day to original doses shown in the protocol of our previous report [6]. This change of doses had provoked unexpected fall of P-LAP activity accompanied by BP aggravation and increased proteinuria ( $3 \mathrm{~g} /$ day). At week $34+5$ day, the doses had been turned back high, but a regular uterine contraction appeared with a tendency of BP to surge. In addition, NST suggested ominous sign of twin A. Therefore, on the next day (week $34+6$ day) we performed elective cesarean section.

A female infant weighing $1684 \mathrm{~g}$ was delivered (Apgar score: 9/10). Twin B was a $400 \mathrm{~g}$ female stillbirth fetus which was macerated without abnormality and two chorionic sacs were confirmed. Weight of the placenta was $470 \mathrm{~g}$. None of pathological abnormality in placenta and cords was found. In puerperium, patient's BP showed 130-150/70-90 $\mathrm{mmHg}$. Proteinuria persisted for nine days. The patient was discharged eighteen days after delivery in noneventful condition. Growth of the newborn had been normal and was discharged from NICU.

\section{Case 2}

In this case, the patient is a 26-year-old (gravid -1, para-1), had severe preeclampsia with underlying complication of non-insulin dependent diabetes mellitus (NIDDM). Since she was diagnosed
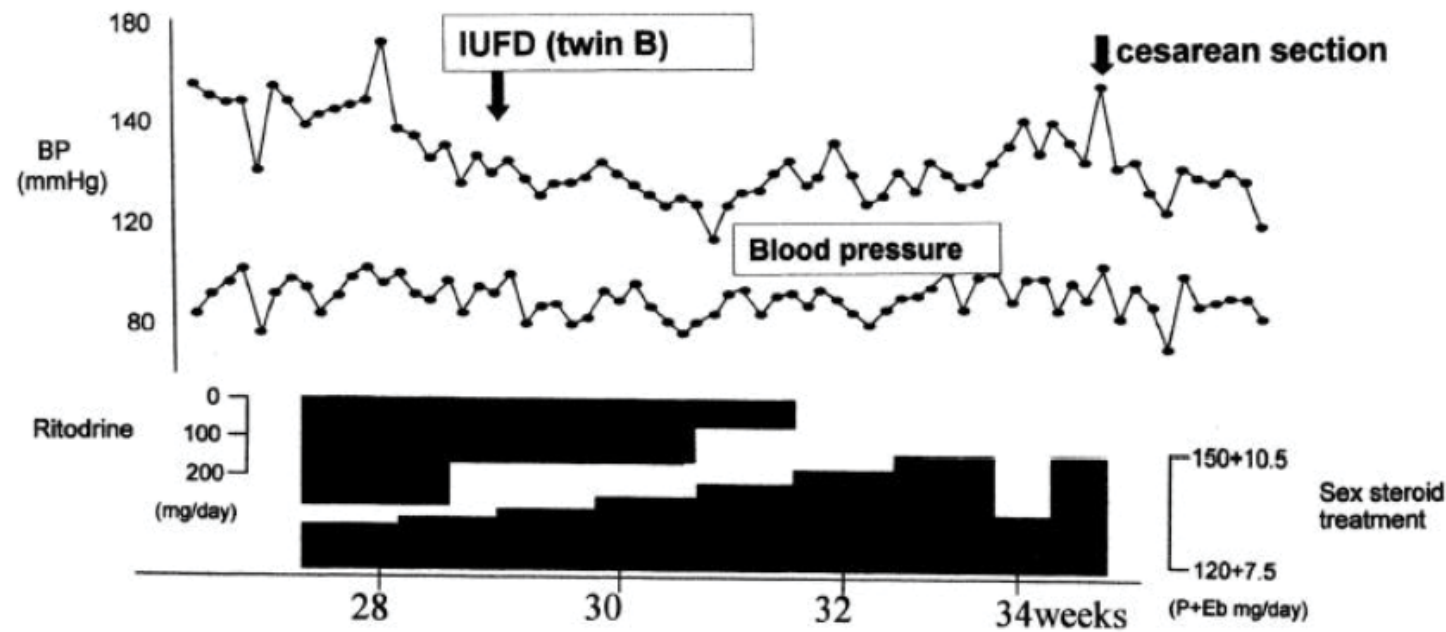

Figure 1: BP decreased temporarily but thereafter had become almost normal and the good condition of twin A was achieved, along with estradiol and progesterone treatment in case 1. 
Citation: Mizutani S, Mizutani E, Iwase A, Shibata K (2018) Treatment for Severe Pregnancy Induced Hypertension and Preterm Labor with Concomitant Administration of Estradiol and Progesterone under Monitoring Aminopeptidases: A Case Report. J Clin Case Rep 8: 1115. doi: 10.4172/2165-7920.10001115
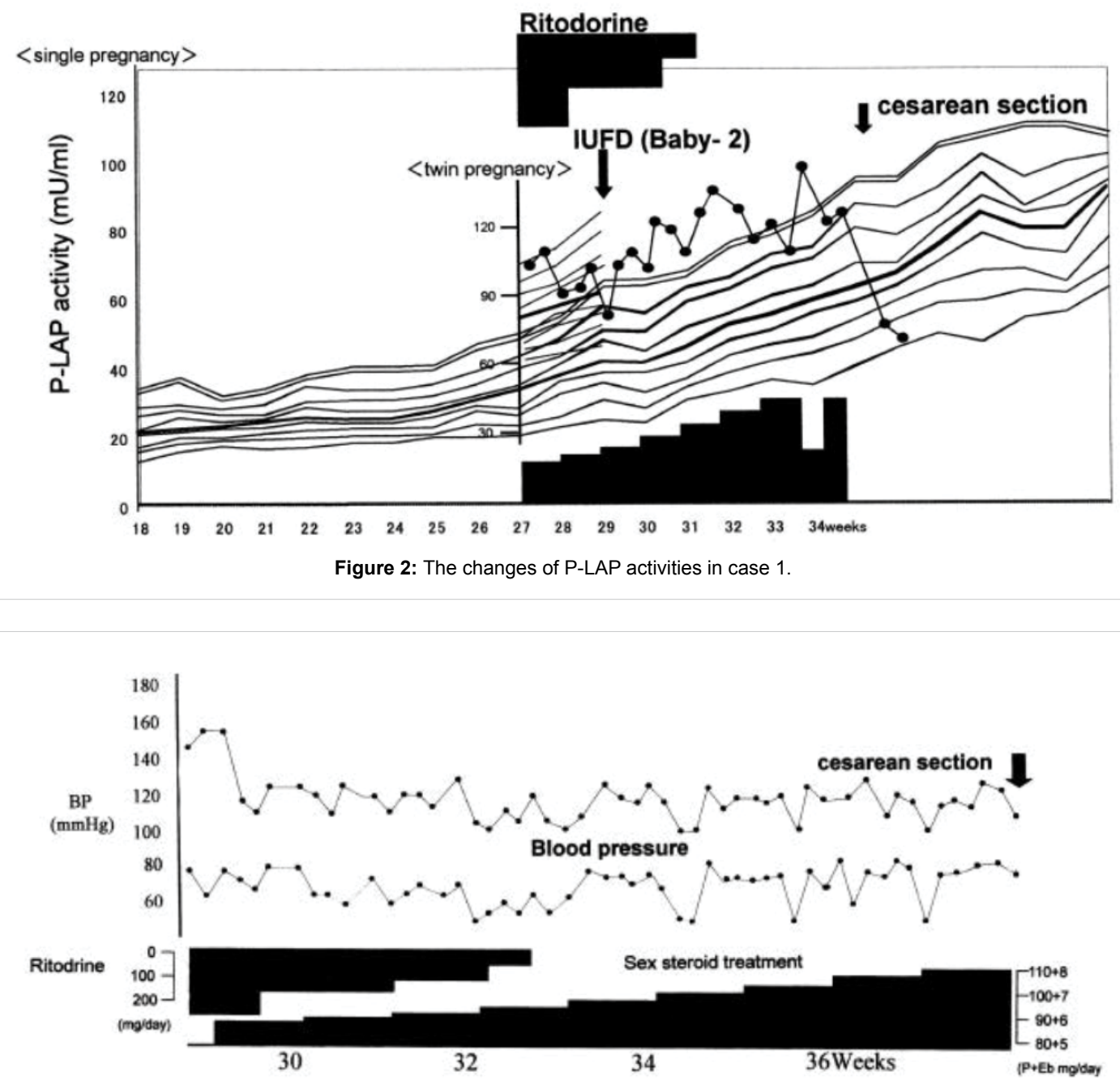

Figure 3: The effects of estradiol and progesterone treatment in case 2.

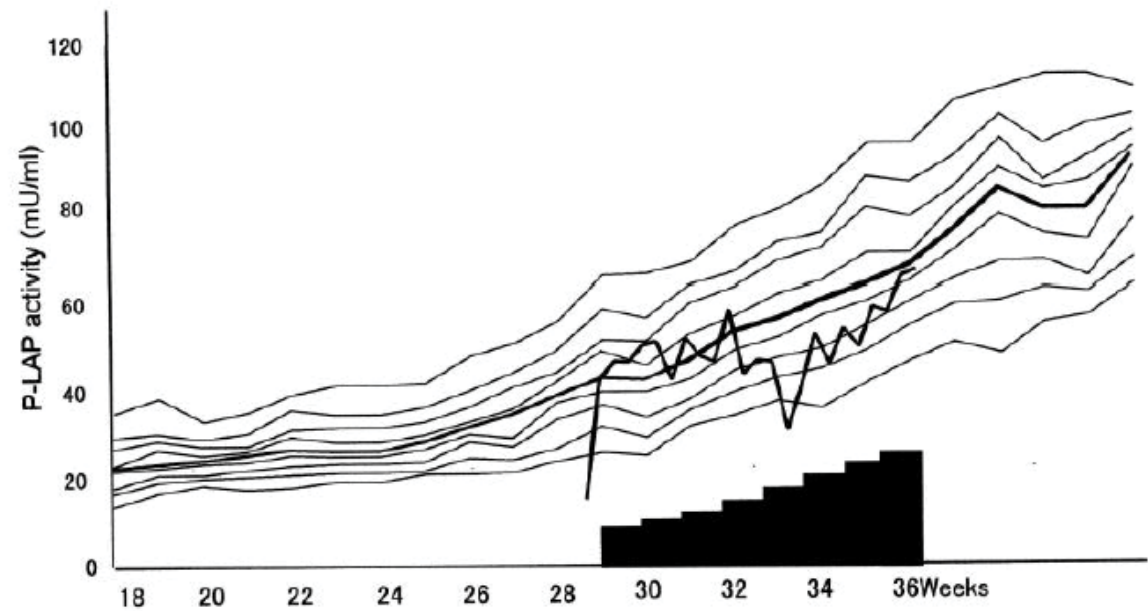

Figure 4: The changes of P-LAP activities in case 2 were shown in black bold line. Before starting sex steroid treatment, P-LAP activity levels had been lower than of normal pregnancy. However, after the treatment initiation, P-LAP activity levels started to increase onto the range of normal pregnancy and kept increased with the treatment, though some fluctuations observed through advancement of gestation. 
for NIDDM at the age of twenty, she had been treated with insulin injections until her pregnancy. In 2003, the patient was admitted to our hospital for mild preeclampsia (BP 155/80 mgHg, proteinuria: 3 $\mathrm{g} /$ day, and edema) complicated by preterm labor (uterine contraction, advanced shortening of uterine cervix: the effacement of uterine cervix reached to about $70 \%$ and dilatation of uterine cervix: around $3 \mathrm{~cm})$. The protruded bag of water from external orifice of the uterus was clearly observed. Ritodorine was administered for the suppression of uterine contraction although the drug is contraindicated to pregnancy with NIDDM. This treatment could induce the increase of blood glucose; therefore, an incremental dose of insulin was required. We proposed the patient our sex steroid treatment accompanied by ritodorine in order to sustain her pregnancy. The patient and her family accepted our treatment approach.

With sex steroid treatment, her symptoms of preeclampsia and preterm labor started to alleviated. Upon observation of symptoms, we were able to decrease the dose of ritodorine gradually and terminate administration at week $32+4$ days. None of antihypertensive agents nor magnesium sulfate were administered (Figure 3 ).

Figure 4 shows the changes of P-LAP activity. Before starting sex steroid treatment, P-LAP activity levels had been lower than of normal pregnancy. However, after the treatment, P-LAP activities started to increase to the range of normal pregnancy and kept increased with the treatment, although fluctuations were observed throughout gestation. The condition of uterine cervix, on the other hand, did not change. At week 37-day premature rapture of membranes occurred and her body temperature $\left(38.2^{\circ} \mathrm{C}\right)$ showed the sign of infection. On the same day, we performed cesarean section. Male infant weighing $2388 \mathrm{~g}$ (Apgar 6/9) was delivered alive. Her puerperium had been in good condition. The newborn was discharged in good condition.

\section{Discussion}

Serum estradiol (E2) and progesterone $(\mathrm{P})$ in normal pregnant women increase with advancing of gestation and reach to about a hundred-fold or more at the point of term compared to preconception. $\mathrm{P}$ is the steroid hormone responsible for carrying over pregnancy by suppressing uterine contraction. Also, the role of E2 is to sustain pregnancy until reaching the timing of initiating labor, but it also has the ability to promote synthesis of P. E2 increases in the uterine blood which ensures adequate supply of oxygen and nutrients to the fetus. In this regard, both $\mathrm{E} 2$ and $\mathrm{P}$ seem to have indispensable roles in carrying over normal term pregnancy [6].

The levels of P-LAP activities under the sex steroid treatment were different between the two cases but they have demonstrated effectiveness and safety of the sex steroid treatment in sustaining a pregnancy. Alleviation of symptoms such as hypertension and uterine contraction were seen as the P-LAP and APA activities increased in maternal sera through advancing of gestation. Changes of P-LAP and APA activities in maternal sera accurately reflected improvement of clinical symptoms $[5,6]$.

As mentioned above, we have shown the fact that both maternal P-LAP and APA activity increase more than normal pregnancy levels in mild preeclampsia, suggesting protective action against the leak of these hormones into the maternal circulation $[2,4]$. In the exacerbation of preeclampsia from mild to severe form, P-LAP activities in maternal sera had abruptly fallen to significantly lower levels than normal pregnancy, indicating the breakdown of the balance between increasing fetal peptide and production of placental Aps $[2,4]$

\section{Conclusion}

Therefore, the difference of the P-LAP levels observed between the two is specific changes of fetal peptide hormone levels before and after the sex steroid treatment. Prognosis of preeclampsia or preterm labor is dependent on P-LAP activities in maternal sera that the pattern what it shows to increase with advancing gestation $[1,5]$. Recently, it was shown that both APA and P-LAP could potentially be safe and effective drugs for medical treatment for preeclampsia and preterm labor [112]. Considering unavailability of safe - to mothers and babies - and effective medication at present, the sex steroid treatment, as proposed in this report, seems worth proceed into comparative clinical studies on these abnormal conditions [6] so that the existing sex steroid hormone preparations could be indicated for preeclampsia and preterm labor for extensive clinical use. Effectiveness of $\mathrm{P}$ and 17-ahydroxyprogesterone caproate (17-P) in preterm labor remains under debate [7]. These mono-progestogen approaches seem devoid of taking account dynamic aspects of estradiol and $\mathrm{P}$ in the placenta. A major defect is the exclusion of estradiol in their protocol [6]. Since the tragedy of diethylstilbestrol [11-13], administration of sex steroid hormones to pregnant women has been averted for a long time until emergence of clinical use in assisted reproductive technology in 1985 [14]. We should not ignore that E2 is an essential element also for the treatment with assisted reproductive technology.

In the near future, the authors expect a pharmaceutical development for both recombinant APA and P-LAP indicated for preeclampsia and preterm labor [1]. This seems the ultimate goal for the effective and safe solution for these abnormalities.

\section{References}

1. Mizutani S, Tsunemi T, Mizutani E, Hattori A, Tsujimoto M, et al. (2013) New insights into the role of aminopeptidases in the treatment for both pre-eclampsia and preterm labor. Exp Opin Investig Drugs 22: 1425-1436

2. Mizutani S, Akiyama H, Kurauchi O, Taira H, Narita O, et al. (1985) Plasma angiotensin I and serum placental leucine aminopeptidase (P-LAP) in preeclampsia. Arch Gyneco 236: 165-1672.

3. Mizutani S, Yamada R, Kurauchi O, Ito Y, Narita O, et al. (1987) Serum aminopeptidase (AAP) in normal pregnancy and pregnancy complicated by pre-eclampsia. Arch Gyneco 240: 27-31.

4. Safwat MA, Mizutani S, Salem HT, Mostafa SA, Itakura A, et al. (1995) Change of placental proteases, which degrade vasoactive peptides, in maternal sear at the onset of preeclampsia. Med Sci Res 22: 83-85.

5. Mizutani S, Kurauchi O, Ito Y, Narita O, Tomoda Y (1989) Positive effect of estradiol and progesterone in severe pre-eclampsia. Exp Clin Endocrinol 92 $161-170$.

6. Mizutani S, Mizutani E (2015) New insights into the role of sex steroid hormones in pregnancy: Possible therapeutic approach by sex steroid hormones for the treatment of both preeclampsia and preterm labor. Exp Clin Endocrinol Diabetes 123: 159-164.

7. Younger JD, Reitman E, Gallos G (2017) Tocolysis: Present and future treatment options. Semin Perinatol 41: 493-504.

8. Jain D, Purohit RC (2014) Review of twin pregnancies with single fetal death Management and fetal outcome. J Obstet Gynecol India 64: 180-183.

9. Mizutani S, Noto H, Inamoto Y, Sakura H, Kawashima Y, et al. (1979) Estimation of placental leucine aminopeptidase in abnormal pregnancy sera. Acta Obst Gynec Jpn 31: 493-498.

10. Mizutani S, Nomura S, Hirose R, Kasugai M, Ishizuka T, et al. (1993) Intrauterine fetal death due to pre-eclampsia which was misdiagnosed to be complicating with hepatitis. Horm Metab Res 25: 187-189.

11. Brown CM, Garovic VD (2011) Mechanism and management of hypertension in pregnant women. Curr Hypertens Res 13: 338-346.

12. Brown CM, Garovic VD (2014) Drug treatment of hypertension in pregnancy. Drugs 74: 283-296. 
Citation: Mizutani S, Mizutani E, Iwase A, Shibata K (2018) Treatment for Severe Pregnancy Induced Hypertension and Preterm Labor with Concomitant Administration of Estradiol and Progesterone under Monitoring Aminopeptidases: A Case Report. J Clin Case Rep 8: 1115. doi: 10.4172/2165-7920.10001115

Page 5 of 5

13. Hoover RN, Hyer M, Pfeiffer RM, Adam E, Bond B, et al. (2011) Adverse health outcomes in women exposed in utero to diethylstilbestrol. N Engl J Med 365: 1304-1314.
14. Macdonough PG (1985) Progesterone therapy: Benefit versus risk. Fertile Steril 44: 13-16. 